\title{
Application on the Problem of the Improved Ant Colony Algorithm on Cloud Computing Scheduling
}

\author{
Zhi-hui Shang ${ }^{1}$, Jian-wei Zhang ${ }^{2}$, Xiao-hua Wang ${ }^{1}$, Hong-jin $\mathrm{Li}^{1}$ and Xu Luo \\ ${ }^{1}$ School of Medical Information Engineering, ZunYi Medical University, \\ Guizhou, 563000, China \\ ${ }^{2}$ Software Engineering College, Zhengzhou University of Light Industry, \\ Zhengzhou, 450002, China \\ zhshang@qq.com
}

\begin{abstract}
In order to solve the problem of long path and long time of addressing the resource scheduling in virtual desktop cloud experiment, we improve the application of ant colony algorithm on the virtual cloud dynamic scheduling problem, which aims at shortening the addressing path and access time. An improved ant colony algorithm cross scheduling strategy is proposed by combining with the restraint function to establish a virtual cloud resource scheduling model so that it can reduce the scheduling time of the resource task being scheduled by the virtual machine. By using the strategy of iterative dynamic programming method to calculate and then combine with the ant colony algorithm, it not only can shorten the addressing time but can enhance the capacity so that it can shorten addressing path length immediately compared with the original ant colony algorithm on scheduling problem. the algorithm has obviously improved a lot on the efficiency of cross scheduling in the cloud resources. The experimental results show that the improved ant colony algorithm has the obvious convergence effects on the path and efficiency of the virtual task resource scheduling.
\end{abstract}

Keywords: Cloud computing; ant colony algorithm; Cross-scheduling; ant colony optimization

\section{Introduction}

Resource scheduling in cloud computing has the advantages of quick access, saving time etc., which is widely accepted and applied. But in the traditional cloud computing algorithm, the static resource scheduling algorithm is in common. However, the static scheduling algorithm cannot meet the requirements of effective scheduling of resources, for example, grid resources can not be met in the problem of optimal allocation. So, in cloud computing, static scheduling cannot be effectively exploited [1]. At present, more and more applications of intelligent optimization algorithms are used in the parallel task of resource scheduling, which greatly improves the efficiency of resource scheduling. Ant colony algorithm (ant colony optimization, ACO) is a probabilistic algorithm that is used to find the optimal path in graph. At the same time, it is also a stochastic search dynamic algorithm, which has high flexibility and robustness in the dynamic scheduling algorithm. Therefore, the ACO algorithm is suitable for solving the parallel task scheduling problem in cloud computing [2].

The cloud computing resource optimization scheduling will be the main research in the future. In order to enhance the resource utilization of cloud computing data Center [3]. Guo Qiyao, etc., in terms of the shortage of cloud computing resources, we propose an effective algorithm combining frog-hop algorithm and ant colony algorithm to introduce

Received (January 4, 2018), Review Result (March 1, 2018), Accepted (April 25, 2018) 
the feedback factor for improving the updating efficiency and convergence rate of pheromone in ant colony algorithm [4]. In order to solve the problem of low precision efficiency of cloud resource scheduling algorithm, Zhao Junpu etc., put forward a genetic ant colony algorithm to optimize the global searching ability, and the genetic ant colony algorithm is used to gain the exact value [5]. Li Chao etc., in the light of the low efficiency of cloud computing resource allocation, proposed a task scheduling algorithm considering time, cost, CPU, memory and bandwidth, etc., [6]. Cui Limei, etc., proposed a hybrid algorithm combining genetic algorithm and ant colony algorithm, which adjusts the optimal path probability problem by setting thresholds for path selection problem in ant colony algorithm [7]. Zhou Mingxiu etc., designed an improved heuristic factor for increasing the effect of the target node on the next node, which avoids enhancing global search capability through local optimization [8]. Nie Qingbin etc., proposed a loadenhanced ant colony algorithm based on time cost through the improvement of pheromone and heuristic information for improving the efficiency of resource scheduling [9]. Li Cheng bing etc., proposed a method of updating pheromone for solving the premature phenomenon of ant colony algorithm in the application of TSP. Thus the practicality and the validity of algorithm will be raised [10].

In order to improve the cloud resource scheduling, the above algorithms mainly focus on the problem of insufficient resource allocation and low efficiency of refinement solution. According to the combination of theory research and the practice, the demand of the access to the virtual resources of the user can be satisfied. But the research method has not started from the resource addressing problem, so this paper focus on the problem of the cloud Computing resource scheduling optimization based on the improved Ant colony algorithm path problem, and proposes an improved ant colony algorithm crossover scheduling strategy, which can optimize the task resource scheduling problem and be more approximate to the real problem.

\section{An Overview of Ant Colony Algorithm}

Ant colony Algorithm (ACO) is a new kind of simulated evolutionary algorithm, which has been proposed by Dorigo M, Maniezzo V and Colorni A in the 1990s to solve some discrete system optimization problems [11-15]. This method obtains a series of good experimental results on the traveling salesman problem, assignment problem, scheduling problem and network routing.

The classical application of ant colony algorithm in scheduling problem can be considered from path problem, which is similar to TSP problem. Task scheduling of Ant Colony Algorithm is to find out the best path from the distance between $\mathrm{n}$ tasks and tasks $[16,17]$. The specific description is as follows:

The first step: Initializing the parameters of ant colony algorithm.

The second step: selecting the next task vertex according to probability, and the calculation of a complete cycle based on the move of each ant.

The third step: Recording the current optimal solution after a cycle, i.e. the optimal path and recording the path length of $\mathrm{M}$ ants, and then calculating the optimal length and keeping the optimal solution.

The fourth step: adjusting the pheromone on the path. updating once according to adjustment of the pheromone concentration when ants finish a complete node process,

The Fifth step: the Tabu table should be emptied and started again from the ACO parameter initialization, if the times setted up haven't been finished. The ant colony algorithm flowchart shown in Figure 1 below is the general algorithm flow, which uses ACO to solve problem. 


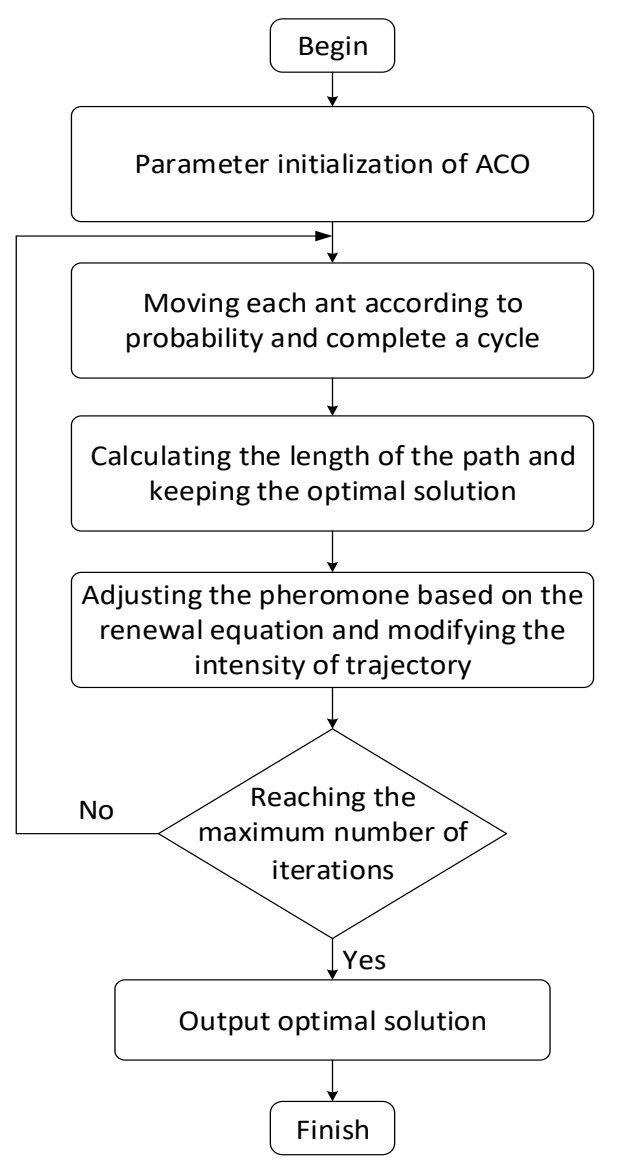

Figure 1. Ant Colony Algorithm Flow

\section{Virtual Cloud Scheduling based on Ant Colony Algorithm}

\subsection{Virtual Cloud Scheduling based on Ant Colony Algorithm}

Ant colony scheduling can solve multiple problems $[18,19]$. This paper takes the virtual cloud lab scheduling as the example. The problem of virtual cloud lab scheduling is usually refers to the operation plan time of each terminal in the start-up process, such as the length of loading, the order of sequence and so on.

In order to quantify the problem, this paper introduces the concept of the system Iteration index $r$ as shown in the formula (1), the system Iteration index $r$ refers to the times that the virtual desktop system is reconstructed (from build to destroy) in a certain type of application scenario. This shows that if the application scenario requires frequent generation and destruction of virtual systems, the system Iteration index $\mathrm{r}$ must be high and vice versa.

$$
r=\frac{\text { Reconstruction times of virtual system }}{\text { Observation time(day) }}
$$

From virtual Cloud Lab, it is known that the virtual cloud desktop is mainly for students ' experiment class and teachers ' daily office use, and the daily Office requires that the virtual cloud desktop should be used only for a particular teacher once generated. If the system is not destroyed or replaced for the requirements of the teacher, it will be used for a long time without being destroyed. Taking the Medical Information Engineering Institute as a example. Assuming that in our school every month 50 teachers have to change the system a time, which is in a high change frequency of the system, then 
the teacher's daily Office System Iteration Index $r=50 / 30=1.7$, this shows that the iterative index of Virtual desktop system is relative low.

In order to solve the above problems, the virtual desktop scheduling problem of the experimental teaching can be classified into the efficient virtual desktop generation. Then the problem can be optimized formally to the following objective functions (2):

$$
\begin{aligned}
\min & \frac{R}{f\left(t_{1}-t_{0}\right)} \\
& \text { s.t. } f\left(t_{1}-t_{0}\right) \geq N \text { and } R \neq a f\left(t_{1}-t_{0}\right)+b
\end{aligned}
$$

Supposing the moment is $t_{0}$, the class hour is $t_{1}$, the number of virtual instances generated before $f\left(t_{1}-t_{0}\right)$ is $t_{1}$, and $R$ is the number of resources consumed by generating $f$ $\left(\mathrm{t}_{1}-\mathrm{t}_{0}\right)$ virtual instances, and $\mathrm{N}$ is the constant representing the minimum requirements for generating virtual instances at $t_{1}$ time, and $R$ and $f\left(t_{1}-t_{0}\right)$ are in nonlinear growth relation.

According to the algorithm analysis design problem, the objective function can be transformed into the problem of finding the best path with the shortest time, so the ant colony algorithm can be improved based of the transformation of problems to find the optimal scheduling time and path in the virtual cloud resource scheduling. Then the optimization of the $\mathrm{f}\left(\mathrm{t}_{1}-\mathrm{t}_{0}\right)$ in the target function can be reached and the efficiency of resource scheduling can be improved.

\subsection{The Objective of Scheduling}

If there are $\mathrm{n}$ kinds of virtual sequence resources, the shortest time to access this resource problem, that is, $X=\min \left(\mathrm{A}_{1}, \mathrm{~A}_{2}, \ldots, \mathrm{A}_{\mathrm{N}}\right), \mathrm{A}$ represents task, $\mathrm{N}$ represents the number of people, $A_{i-1}$ represents A working i-1 tasks, the objective function is the best completion time of minimization, usually $\min \left(\mathrm{A}_{1}, \mathrm{~A}_{2}, \ldots, \mathrm{A}_{\mathrm{N}}\right)=\min \left\{\mathrm{T}_{\mathrm{i}}\right\}, \mathrm{i}=1,2, \ldots, \mathrm{N} . \mathrm{T}_{\mathrm{i}}$ is the time used for loading the first task, which is T. According to description of the above problem, the ways of loading all the resources are $\left(\sum_{i=1}^{n} n p_{i}\right) !$. The general load tasks are given in the virtual cloud resource scheduling problem, and the total number of job scheduling is generally $\left(\frac{1}{w p_{1} !} \frac{1}{w p_{2} !} \cdots \frac{1}{w p_{n} !}\right)\left(\sum_{i=1}^{n} n p_{i}\right) !$. But with the upgrade and expansion of the application of large data, service cluster and resource scheduling tasks are increasing and effective utilization of resources is improving. Therefore, being able to access to effective resources efficiently and quickly become the focus of future research. The main task of resource scheduling algorithm is to meet the optimal or effective loading sequence in the shortest time[20,21].

\section{The Before and Improved Ant Colony Algorithm}

\subsection{Implementation Steps of Ant Colony Algorithm before Improvement}

In actual biological systems, ants communicate with each other by releasing secretions in the process of finding food, which are often called pheromone. Ant colony algorithm mainly solves TSP (Traveling Salesman Problem) problem. TSP problem is solved mainly by combining the sections that ants like. The way of solving the problem of TSP is based on the idea of the ant colony algorithm, which transforms it as ant colony algorithm solve the problem of virtual cloud dispatch.

First of all, taking TSP as an example, in the process of solving the problem, the ant colony algorithm uses $\mathrm{m}, \eta_{i j}, \tau_{i j}, \Delta \tau_{i j}^{k}$ as the parameter variables, in which $\mathrm{m}$ represents the virtual resource quantity, $\eta_{i j}$ represents the visibility of the curvature path $(i \rightarrow j)$ for 
the virtual job to be invoked, i.e., the $1 / \mathrm{d}_{\mathrm{ij}}$, represents the track intensity for the side arc (i $\rightarrow \mathrm{j}$ ), and $\Delta \tau_{i j}^{k}$ represents the number of pheromone of unit length of job $\mathrm{K}$ in the path $\mathrm{i} \rightarrow \mathrm{j}$. According to different values of $\Delta \tau_{i j}^{k}$, different types of ant colony algorithm can be formed. But the most basic ant colony algorithm is shown in formula (3).

$$
\Delta \tau_{i j}^{k}=\left\{\begin{array}{l}
C / M_{k} \\
0, \text { others }
\end{array}\right.
$$

if $(\mathrm{i}, \mathrm{j})$ is on the best path, and $M_{k}$ is the target function value, then $l_{i j}^{k}$ represents the transfer probability of job $\mathrm{k}$, which is a proportional function with $\eta_{i j}$ and $\tau_{i j}$. $\mathrm{J}$ is the node that has not yet been reached. The update equation for trajectory strength is shown in the formula (4).

$$
\tau_{i j 1}=\rho \tau_{i j 2}+\sum_{\mathrm{k}} \square \tau_{i j}^{k}
$$

Second, $\tau_{i j 1}$ represents the visibility of the newest side radian path $(i \rightarrow j) . \tau_{i j 2}$ represents the visibility of the previous side Radian path $(\mathrm{i} \rightarrow \mathrm{j}) . \alpha$ is the relative importance parameter of the orbit and the scope is $\alpha \geq 0 . \beta$ is the importance of visibility parameters, whose range is $\beta \geq 0$. $\rho$ represents the persistence parameter of the trajectory, whose range is $0 \leq \rho<1$, and $1-\rho$ represents the attenuation of the trajectory. $C$ is a path trajectory constant that is left after the scheduling of virtual resource.

Finally, if the number of scheduling task of virtual resources based on ACO does not meet our pre-set parameters, the tabu table where the load-path is stored should be emptied and started the calculation again from the parameter initialization set by ACO. The above algorithm is the general algorithm flow of ACO for solving the resource scheduling problem before the improvement.

\subsection{The Realization Steps of Improved Ant Colony Algorithm}

Based on the job scheduling research and application of ant colony algorithm in Virtual Cloud resource environment, this paper proposes a new algorithm strategy, which is ant colony algorithm cyclic preloading strategy, and resource scheduling problem is a typical shortest path solving problem, that is, NP difficult problem. In practical application, the solution that the ant colony algorithm converges to is usually the local optimal solution, not the global optimal solution. Secondly, the global search capability based on ant colony algorithm is strong, but the local search ability is weak, so some auxiliary skills are often embedded according to the requirement.

Therefore, based on ant colony algorithm, the technique of dynamic programming algorithm is used to realize cross iterative solution [18]. In this paper, an improved ant colony algorithm scheduling strategy is proposed, which is a cross scheduling strategy. In the aspect of virtual cloud resource scheduling, the algorithm has obvious convergence effect on the scheduling time of task assignment and the scheduling path of task assignment.

First, assuming $\mathrm{V}$ as a subset of the set $\{2,3, \ldots, \mathrm{m}\}, k \in V . \mathrm{M}(\mathrm{v}, \mathrm{k})$ represents the point of traversing $\mathrm{v}$ from the first job, terminating and recording the optimal path length after the $\mathrm{K}$ point reached. When $|\mathrm{V}|=1, \mathrm{M}(\{\mathrm{k}\}, \mathrm{k})=\mathrm{d}_{1, \mathrm{k}}, \mathrm{k}=2,3, \ldots, \mathrm{m}$ when $|\mathrm{V}|>1$, according to the principle of finding the optimal solution, the dynamic programming equation of the Dynamic scheduling virtual task job can be written as shown in the formula (5). 


$$
M(V, k)=\left\{\begin{array}{l}
\min \left\{M(V-\{k\}, l)+d_{l k}\right\}, j \in V \\
0, j \notin V
\end{array}\right.
$$

Secondly, in order to satisfy the reduction of the path weights of the optimal load task job, the optimal solution can be solved by the recursive iterative solution of the equation (5). And the solution loaded into the ant colony algorithm cycle queue can be transformed the optimal dispatch path optimization solution. Because the dynamic programming problem mainly solves the big problem of combinatorial number, the method is usually not used in solving the problem of small scale. Based on the analysis of the performance of resource scheduling, the selection of the value of $\alpha, \beta, \rho$, and without the consideration of the value selection of the initial pheromone $\mathrm{C}$, it is difficult to realize the global optimization function to prevent the path of the task job loading from the local optimal solution.

Assuming $\tau_{i j}(0)=\mathrm{C}$, and $\mathrm{C}$ is a constant. Job Resource $\mathrm{K}$ releases pheromone according to the iterative order of optimal value scheduling in ant colony algorithm, and then determines the direction of job resources being dispatched and transferred according to the release amount of pheromone. ${ }^{\tau_{i j}}(t)$ represents the probability that the job resource $\mathrm{K}$ is transferred from the dispatched position $\mathrm{i}$ to the $\mathrm{j}$, i.e., the size of the trajectory intensity.

$$
p_{i j}^{k}=\left\{\begin{array}{l}
\frac{\left[\tau_{i j}(t)\right]^{\alpha} \bullet\left[\eta_{i j}(t)\right]^{\beta}}{\sum\left[\tau_{i s}(t)\right]^{\alpha} \bullet\left[\eta_{i s}(t)\right]^{\beta}}, j \in \text { allowed }_{k} \\
S \in \text { allowed }_{k} \\
0, \text { others }
\end{array}\right.
$$

$\eta_{i j}$ represents the visibility of the curved path $(\mathrm{i} \rightarrow \mathrm{j})$ to which the virtual job is invoked.

It is known from the heuristic algorithm that the general value is

$$
\eta_{i j}(t)=1 / d_{i j} \text {. }
$$
$d_{i j}$ represents the job scheduling distance from $\mathrm{i}$ to the j. $\alpha, \beta, \rho$ are also introduced above, in which, $\alpha$ represents the relatively important parameters of orbit, ranging for $\alpha \geq 0$, and $\beta$ represents the importance of visibility parameters, ranging for $\beta \geq 0$, and $\rho$ represents the persistence parameter of the trajectory, ranging for $0 \leq \rho<$ 1 , and ${ }^{1-} \rho$ represents the attenuation of the trajectory. Task Resource $\mathrm{K}$ dispatched to the next node marked as allowed $_{k}$ in the note. In the formula (6), the shortest path length will update once dynamically when virtual resources addressed to each node, and the updated data will be saved into a two-dimensional table.

Finally, the formula (7) is used to assign the parameter, 1- $\rho$ indicates the attenuation of the trajectory, and different time represents different attenuation degree. $t+n$ represents the location of the virtual machine to the new node, and the $\mathrm{T}$ node indicates the first time of leaving the virtual machine. $\square \tau_{i j}$ Represents the change of pheromone in the process of task resource scheduling, and $\mathrm{M}$ is the number of resource task, so $\square \tau_{i j}$ cannot be much smaller than the quantity of $\mathrm{C}$, otherwise the research efficiency of the 
problem is not obvious; However, if $\square \tau_{i j}$ is larger than the quantity of constant $\mathrm{C}$ is also not conducive to the study of the problem, which will lead to the exceed expectations of impact of the problem when previous tasks are scheduled, resulting in the local circulation optimal state. Therefore, the value of $\square \tau_{i j}$ should be reasonable. According to previous research and calculation, it is found that the when the value of $\square \tau_{i j}$ is $\frac{1}{5} C$, which will be more appropriate and have better convergence effect.

$$
\begin{gathered}
\tau_{i j}(t+n)=(1-\rho) \bullet \tau_{i j}(t)+\square \tau_{i j} \\
\Delta \tau_{\mathrm{ij}}=\sum_{k=1}^{m} \square \tau_{i j}^{k}
\end{gathered}
$$

So, the research and application of ant colony algorithm in job scheduling is compared with the cyclic loading strategy combined with ant colony algorithm by iterative algorithm of dynamic programming in this paper. The best optimal dispatch path is taken from two studies and then the optimal solution retained from the study is compared with the optimal solution in this study.

\section{The Analysis and Experimental Results of Simulation Environment}

\subsection{The Establishment of Simulation Platform}

The simulation environment includes hardware and software. The test hardware and software of building a platform mainly are: CPU Intel (R) Pentium (R) G2030 3.00GHz, microsoft Windows 2007 4GRAM, and the simulation platforms are two platforms achieved by the simulation results of the simulation cloud platform Cloudsim and MATLAB R2012A.Compareing the strategy of the combination of ant colony algorithm and dynamic programming algorithm with the original ant colony algorithm.

Due to the components of simulation platform Cloudsim are open source, so the second development can be implemented base on the combination of architecture of Cloudsim software and structure components of Simjava, Gridsim, Cloudsim, Usercode. If the mapping realization method is modified by Simjava, Gridsim, Cloudsim etc. from Cloudlet to the virtual machine (VM), then the target value will be achieved through the mutation operation of cross_a, cross_b, cross_c, cross_d. Thus, according to the Cloudsim simulation platform, first, the Gridsim library should be initialized, and then the data center made up of one or more machine should be created. According to agent broker and the creation of virtual machine, finally, the simulation will be started through the cloud task. Combining with MATLAB simulation results, the optimal routing length is computed and the time of the best path is recorded. In the end, the total optimal scheduling path of several processor will be calculated, which is the global optimal scheduling value.

The previous work is to create virtual machines by virtual cloud platform and set the number of tasks. Building the virtual environment simulation platform through using the network hardware and software resources and then settings the compilation of ant class, and finally verifying the experiment through the task of loading algorithm joining in the virtual cloud experimental environment platform.

The setting of parameters are as follows: According to previous research, setting up 30 virtual cloud terminal server cluster, heuristic factor is invariant. Ordering $\alpha=1.5$, the expectation value of heuristic factor is $\beta=1.8$, so the task resource quantity $m=30$, setting the pheromone's volatilization factor as $\rho=0.5$. Through the parameter setting and the experiment verification of virtual cloud Platform, comparing the hybrid loading strategy 
combining iterative solution of dynamic algorithm and ant colony algorithm with the original ant colony algorithm strategy.

According to the scheduling tasks number of 30 virtual machine, the two dimensional simulation path weights of each virtual machine are randomly given, in which the range of $\mathrm{X}, \mathrm{Y}$ is: $0 \leq X \leq 100,0 \leq Y \leq 100$, as shown in Table 1 below.

Table 1. The Two-Dimensional Weight of Virtual Tasks

\begin{tabular}{|c|c|}
\hline $\begin{array}{l}\text { value of the } \\
\text { weight } \\
\text { coordinate }\end{array}$ & test data \\
\hline $\mathrm{X}$ & $\begin{array}{lllllllllllllll}23 & 12 & 56 & 3 & 76 & 6 & 85 & 71 & 21 & 32 & 44 & 18 & 21 & 67 & 91 \\
79 & 54 & 2 & 68 & 74 & 27 & 54 & 16 & 19 & 75 & 28 & 94 & 69 & 35 & 10\end{array}$ \\
\hline $\mathrm{Y}$ & $\begin{array}{lllllllllllllll}6 & 31 & 69 & 21 & 8 & 73 & 79 & 43 & 62 & 55 & 14 & 32 & 88 & 16 & 8 \\
42 & 71 & 38 & 21 & 7 & 32 & 93 & 5 & 34 & 17 & 34 & 68 & 23 & 26 & 84\end{array}$ \\
\hline
\end{tabular}

\subsection{The Comparison and Analysis of Simulation Results}

The results of simulation experiment shows that the optimal dispatch path weights of the ant colony algorithm based on the original research of 30 job tasks need to be dispatched by MATLAB, and compared with the optimal addressing path calculated by the previous research. Then a two-dimensional path map of optimal dispatch is obtained. According to MATLAB, the path weighted value of the optimal path of the ACO scheduling is 461.8416, and the optimal path map of ACO cross scheduling before improved is shown in Figure 2 below.

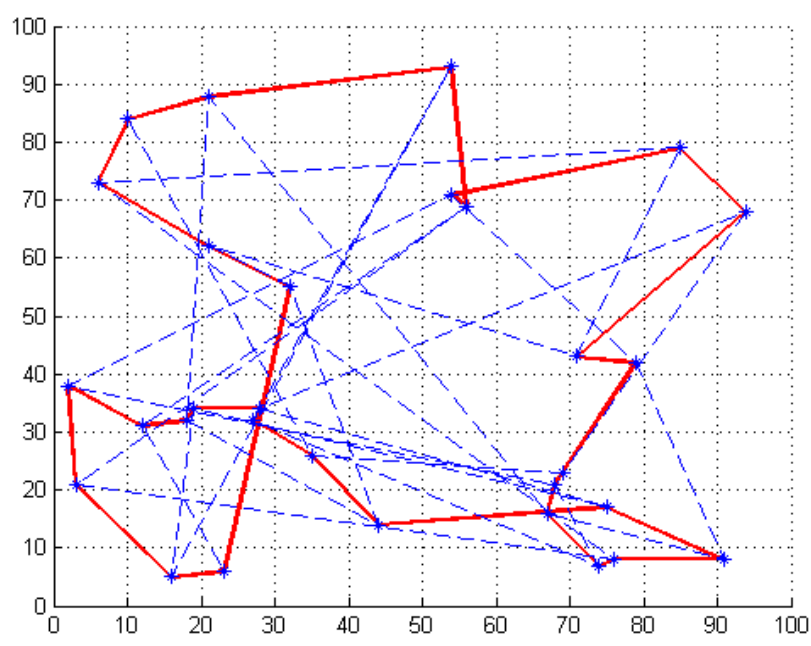

\section{Figure 2. The Optimal Path of Original ACO Scheduling}

First, according to the original research, the best node in the ant colony algorithm is found out and the cyclic operation is done through a the job resource scheduling. Then, the overall relative optimal solution will be obtained by the cycle of the local optimal. However, as for the current algorithm strategy, the assistant algorithm technique should be used. And the optimal route node is iteratively obtained through the dynamic programming algorithm, and then the optimal node is selectively adjusted. Finally, according to the optimal node selected at present, the local optimal state is shifted to a better local optimal state by Ant colony algorithm. Thus a global relative optimal state 
will be achieved. The optimal path weight value of the improved ACO crossover scheduling strategy is calculated, and the optimal path weighted value is 458.2685 . the optimal path map for improved ACO Cross scheduling is shown in Figure 3.

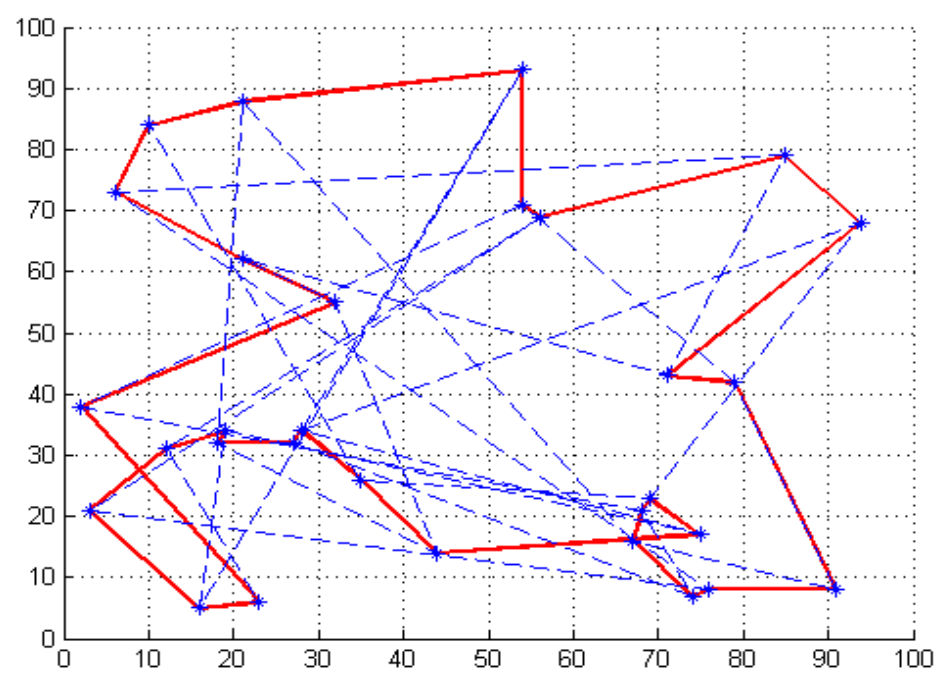

Figure 3. The Optimal Path of Improved ACO Scheduling

\subsection{The Value Comparison of Optimal Scheduling Path between Previous and Improved Algorithm}

In this paper, taking the virtual machine scheduling resource responding to 30 tasks as an example, in the environment of virtual cloud resource, virtual teaching in laboratory is regarded as the simulation. When the number of task assignments is very small, the sum of weighted optimal paths of the improved ACO and the original ant colony algorithm is not obvious, because the proposed strategy is suitable for the larger number of combinatorial problems. Therefore, in the case of a large number of service clusters and under the premise that the set of simulation data is generally no more than 500 , the comparison between the two strategies is obvious, and the scheduling effect will be slightly improved.

When setting different parameters for task assignment, it is found that the improved ant colony algorithm scheduling strategy is more obvious in virtual cloud scheduling compared the two methods with each other. So setting up the same number of different data in the two virtual jobs, calculating the best scheduling path and comparing with each other through the original ant colony algorithm and improved dynamic cross scheduling strategy. the change of vale is $\Delta \mathrm{L}$. and the comparison table of setting the parameter is shown in Table 2 below.

Table 2. Change Value of Best Path

\begin{tabular}{c|llllll}
\hline number of tasks & 0 & 10 & 30 & 100 & 200 & 500 \\
\hline$\Delta \mathrm{L}$ & 0 & 0.93 & 3.57 & 7.26 & 11.05 & 12.07 \\
\hline
\end{tabular}

Comparing of the optimal scheduling path changes through experiments. It can be seen in the table when the resource volume is not more than 10, the changes of optimal addressing path generally not more than 1 . However, with the increasing of resources, the amount of changes has increased. The path change of the improved ACO scheduling strategy is getting more and more large, but it may be approximated to a certain value. 
Based on the comparison between the original ACO scheduling algorithm and the improved cross segment strategy algorithm, it is found that the improved optimal routing path has obvious convergence effect. According to the different task resources, the sum of the best addressing paths corresponding to two strategies are calculated out. Then a column contrast chart of weight value is made and the results are not significant, which is shown in Figure 4 below. However, by means of making the difference of weights, it is found that the optimal path difference of the same number of task resources is significantly shortened, and the shortening distance tends to a certain value, as shown in Figure 5 below.

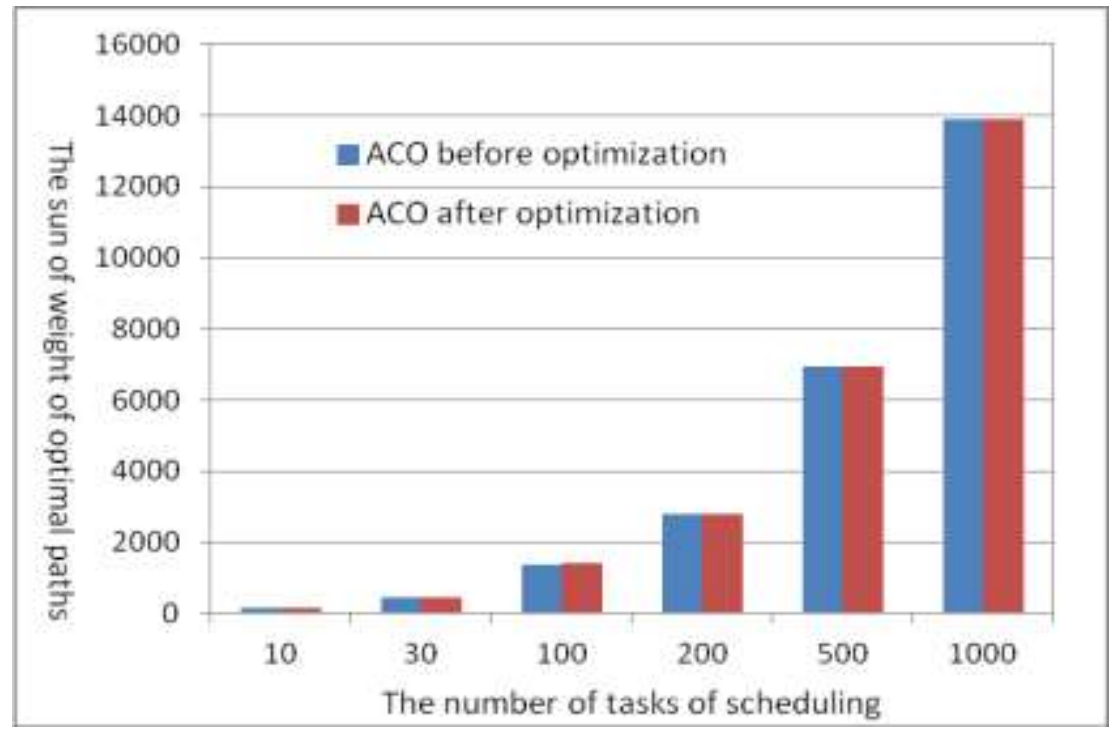

Figure 4. The Contrast Graph of the Sum of Weight

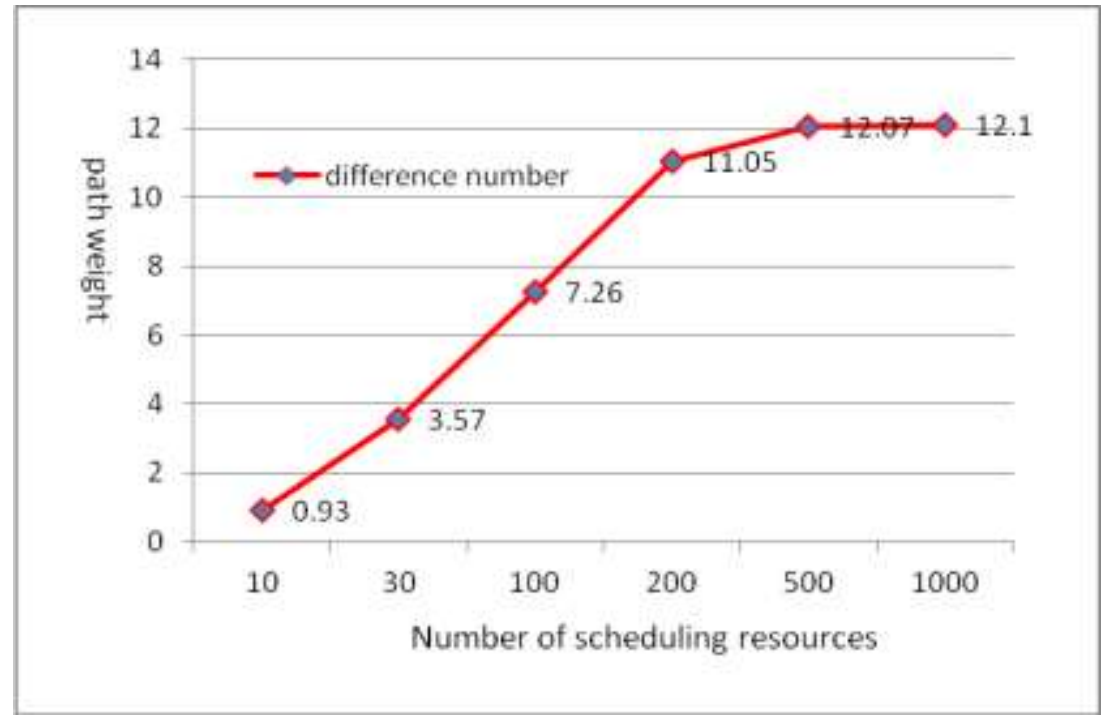

Figure 5. The Contrast Graph of Difference Value

\subsection{The Conclusion of Experiments}

Summing up the above experimental results, comparing the optimal scheduling path summation of the original ACO with the iterative solution of the dynamic programming method combing the cross scheduling strategy of ACO, and then we make a comparative analysis of the shortest path and give a further analysis of the shortest path weights and 
sizes of the two strategies. By visualizing the histogram and graph of graphs, there is no obvious difference between the optimal addressing scheduling path weights of two strategies for different task resources, but after making difference between the ACO before improved and the improved ACO in the optimal dispatch path weights, it is found that the variance of the weighted value of the task quantity increases obviously, and may reach to a certain optimal value. Therefore, the improved strategy method improves the optimal addressing and efficiency, and further illustrates the effectiveness of the algorithm in virtual resource scheduling.

\section{Conclusions}

In this paper, a new strategy method is proposed for dynamic programming to decompose a number of child problems, and then the iterative solution of the sub problem and the Ant colony algorithm is used to solve the problem. Comparing the previous algorithm strategy with the new strategy method proposed in this paper, the experimental results show that the method of cross scheduling of dynamic programming combining ant colony algorithm has a little convergence effect on the path problem. This method not only improves the local optimal state, but also shortens the task processing time and scheduling time, and optimizes the optimal scheduling path of task resources, which has a great reference value for the future virtual resource scheduling problem. But in this paper the research is carried out mainly according to the number of the college server cluster, which is in the control of 1000 units. Thus, it is obvious that in the next large data environment, the large virtual cluster in the scheduling path addressing research needs further improvement and optimization. Therefore, the research work in the future mainly focus on the path addressing problem under large-scale service cluster, as well as resource allocation, service matching degree and other issues.

\section{Acknowledgements}

This work is Supported by 2016 Outstanding Innovation Science and Technology Youth Project of the Innovation Scientists and Technicians Plan of Henan Province: research of the macro scenario fitting routing technology centering on content. 2016 National Natural Science Foundation Project: Research of Self-Adaptive Routing based on Macro-Context Fitting in Content-Centric Networks(no.61672471) and The research is sponsored by National Natural Science Foundation(NNSF) of China under Grant (no.61463053). Science research start-up funds project for master of Natural Sciences of Zunyi Medical University in 2017.

\section{References}

[1] P. Mu, "Construction of Intelligent Teaching Environment in University Based on Internet of Things", Cloud Technology and Big Data.Journal of Chongqing Normal University (Natural Science), vol. 34, no. 5, (2017), pp. 81-86.

[2] Q. Y. Lu and X. M. Zhang, "Adaptive Hybrid Mutation Gravitational Search Algorithm”, Journal of Chongqing Normal University (Natural Science), vol. 34, no. 3, (2017), pp. 85-90.

[3] M. Dorigo and C. Blum, "Ant colony optimization theory: A survey", International Journal of Productivity \& Performance Management, vol. 59, no. 2, (2010), pp. 811-828.

[4] Q. Y. Guo and F. D. Zhu, "Cloud Computing Resource Scheduling Algorithm Based on Ant Colony Algorithm and Leapfrog Algo", Bulletin of Science and Technology, vol. 33, no. 5, (2017), pp. 167-170.

[5] J. P. Zhao, J. Y. Yin and T. B. Jin, "Application of genetic ant colony algorithm in cloud computing resource scheduling”, Computer Engineering and Design, vol. 38, no. 3, (2017), pp. 693-697.

[6] C. Li, B. R. Dai, Z. Z. Kuang, X. L. Wu and R. Q. Sun, "Research on Task Scheduling with Multiple Constraints Based on Genetic Algorithm in Cloud Computing", Journal of Chinese Computer Systems, vol. 38, no. 9, (2017), pp. 1945-1949.

[7] L M. Cui, "Research of Resource Scheduling Based on ACA-GA in the Cloud Computer", Bulletin of Science and Technology, vol. 32, no. 9, (2016), pp. 149-153. 
[8] M. X. Zhou, K. Cheng and Z. X. Wang, "Improved Ant Colony Algorithm With Planning of Dynamic Path", Computer Science, vol. 40, no. 1, (2013), pp. 314-316.

[9] Q. B. Nie, T. Cai and N. Wang, "Aoolication of improved ant colony algorithm in resource allocation of cloud computing”, Computer Engineering and Design, vol. 37, no. 8, (2016), pp. 2016-2020.

[10] C. B. Li, R. X. Guo and M. Li, "Application of improved ant colony algorithm in travelling salesman problem”, Journal of Computer Applications, vol. 34, no. S1, (2014), pp. 83-85.

[11] S. M. Abdulhamid, S. H. H. Madni, M. S. Abd Latiff, M. Abdullahi and M. J. Usman," Cloud Workloads(HPC2N Dataset)", Figshare. Retrieved: 11 25, (GMT), (2017) April 18.

[12] S. H. H. Madni, M. S. A. Latiff and Y. Coulibaly, "Resource scheduling for infrastructure as a service (IaaS) in cloud computing: Challenges and opportunities", Journal of Network and Computer Applications, vol. 68, (2016), pp. 173-200.

[13] S. Devipriya and C. Ramesh, "Improved max-min heuristic model for task scheduling in cloud", Green Computing, Communication and Conservation of Energy (ICGCE), 2013 International Conference on IEEE, (2013).

[14] S. Jamali, F. Alizadeh and S. Sadeqi, "Task Scheduling in Cloud Computing Using Particle Swarm Optimization", The Book of Extended Abstracts, (2016), pp. 192.

[15] P. Akilandeswari and H. Srimathi, "Survey and analysis on Task scheduling in Cloud environment", Indian Journal of Science and Technology, vol. 9, no. 37, (2016), pp. 1-6.

[16] K. R. Ku-Mahamud, A. M. Din and H. J. A. Nasir, "Enhancement of Ant Colony Optimization for Grid Load Balancing", European Journal of Scientific Research, vol. 64, no. 1, (2011), pp. 42-50.

[17] Z. H. Shang, J. W. Zhang and Z. Y. Cai, "Research of Scheduling Method Based on Dynamic Ant Colony Genetic Algorithm in Cloud Environment", Automation \& Instrumentation, vol. 31, no. 11, (2016), pp. 11-15.

[18] F. Sharifi Milani and A. Habibizad Navin, "Multi-Objective Task Scheduling in the Cloud Computing based on the Patrice Swarm Optimization", International Journal of Information Technology and Computer Science (IJITCS), vol. 7, no. 5, (2015), pp. 61.

[19] M. Malawski, G. Juve, E. Deelman and J. Nabrzyski, "Cost-and deadline-constrained provisioning for scientific workflow ensembles in IaaS clouds", Proc. Int. Conf. High Performance Comput. Netw. Storage Anal, no. 22, (2012).

[20] M. Ali, S. U. Khan and A. V. Vasilakos, "Security in cloud computing: Opportunities and challenges", Inf. Sci., vol. 305, (2015), pp. 357-383.

[21] M. Tanaka and O. Tatebe, "Workflow scheduling to minimize data movement using multi-constraint graph partitioning", in Proc. 12th IEEE/ACM Int. Symp. Cluster Cloud Grid Comput., (2012) May, pp. $65-72$.

\section{Authors}

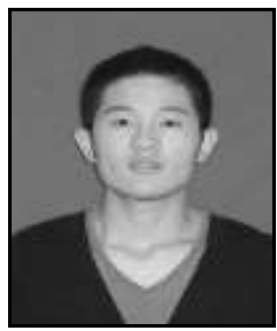

Zhi-hui Shang, born in November, 1989, Henan, grades: Assistant at ZunYi Medical University, China University studies: Master's degree from Zhengzhou University of Light Industry in China, Scientific interest: Cloud computing, broadband information network and network security.

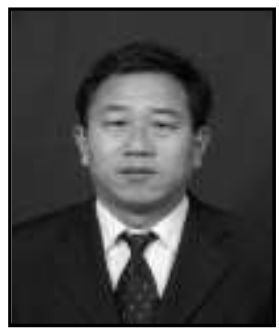

Jian-wei Zhang, born in April, 1971, Henan, P R china Current position, grades: Professor at Zhengzhou University of Light Industry, China University studies: PhD degree from The PLA Information Engineering University in China Scientific interest: broadband information network and network security. 